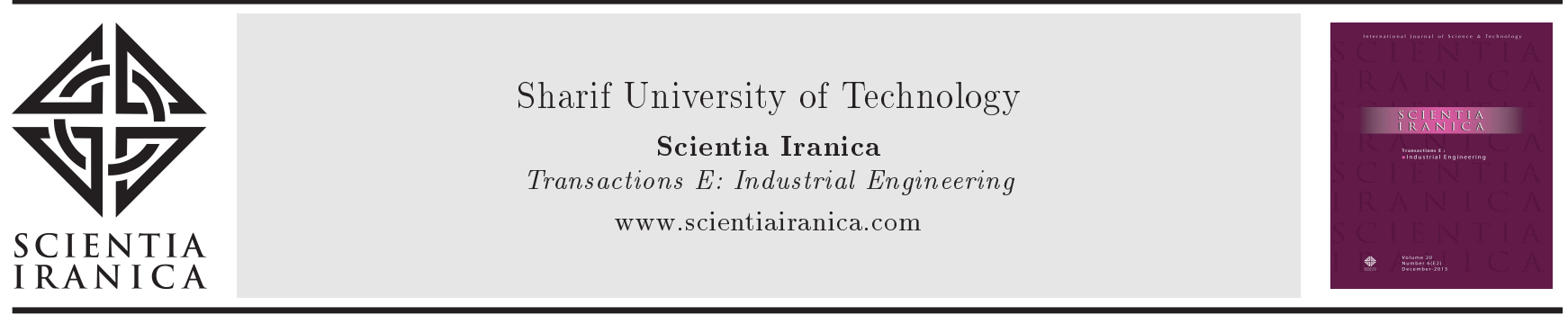

\title{
Supply chain coordination via a two-way cooperative advertising contract considering competing retailers
}

\author{
S. Alaei and M. Setak* \\ Department of Industrial Engineering, K.N. Toosi University of Technology, Tehran, Iran.
}

Received 7 December 2014; received in revised form 20 July 2015; accepted 15 September 2015

KEYWORDS
Cooperative
advertising;
Coordination;
Contract design;
Competing retailers;
Game theory.

\section{Introduction}

With the lack of cooperation, supply chain participants make their decisions independently and noncooperatively. This may lead to channel inefficiency. An important issue in supply chain management is to design mechanisms that can align the individual objectives and coordinate the activities such as productiondistribution, production-inventory, pricing-advertising, etc.

There is a variety of coordination mechanisms in the literature; for example, wholesale price contract [1], two-part tariff [2], revenue sharing [3], quantity discount [4,5], sales rebate return [6], etc. For more information, the readers may refer to Arshinder et al. [7] for a comprehensive review of supply chain coordination mechanisms.

\footnotetext{
*. Corresponding author. Tel.: +98 2184063373 ;

Fax: +982188674858

E-mail address: setak@kntu.ac.ir (M. Setak)
}

Vertical cooperative advertising introduced by Berger [8] is a cost sharing mechanism which manufacturers offer to motivate their retailers to invest more in advertising. Advertising is divided into national and local efforts. The manufacturer's national advertising is budgeted for expanding the product's brand image. However, retailers' local effort focuses on customers' short-term buying behavior [9]. In a traditional vertical cooperative advertising, the manufacturer shares a fraction of retailers' local advertising costs (i.e., the manufacturer's participation rate).

Cooperative advertising is one of the most effective methods of advertising and marketing. It has been highly promoted in today's marketing practices. Companies such as Apple [10] and IBM [11] have benefited from this program. Small online co-op advertising does exist particularly in automotive and durable goods [12]. The interested readers may refer to "Co-op Advertising Programs Sourcebook" which lists thousands of available co-op programs in 52 product classifications. The categories range from agricultural 
products to toys.

Co-op advertising has been discussed in the literature, where the participation rates are exogenously given $[13,14]$. However, the supply chain members may want to optimize the participation rates as discussed by Zhang et al. [15]. Optimizing the participation rates in the cooperative advertising program via endogenous setting may hurt the whole supply chain efficiency. When one of the participants in the supply chain optimizes a specific variable, the other participants' objectives may be ignored; accordingly, the whole channels objectives may be ignored [16]. In this paper, we investigate this feature by assuming both endogenous and exogenous scenarios and investigate the effect of each parameter setting on supply chain efficiency.

Almost all of the research articles on cooperative advertising focus on one-way subsidy contract where only the manufacturer shares the retailer's advertising costs. There are only three articles which consider bilateral participation in the cooperative advertising program [13-15]. However, their models do not consider retailers' competition in the downstream echelon of the supply chain. Although, there are some research papers in the literature on cooperative advertising in a duopoly market [17-20], however, to the best of our knowledge, the effect of a two-way subsidy contract has not been considered yet.

The objectives of the paper are: (a) to investigate the potential benefits of the two-way subsidy contract comparing with the traditional one-way contract; (b) to explore the differences between endogenous parameter setting and exogenously determined parameters setting; (c) to investigate the effect of competition between the retailers on supply chain efficiency. So, the main contributions of this research are as follows: The model compares the two-way subsidy strategy with the traditional one-way strategy to explore its potential advantages. Also, the competition effect of retailers on the channel efficiency is examined.

We study a supply chain with a single manufacturer and two identical retailers. Participants located in echelons (i.e., the manufacturer in the upstream and two retailers in the downstream), play a Stackelberg game. Moreover, in downstream echelon of the channel, retailers obey either collusion or Stackelberg behavior. A two-way subsidy strategy for channel coordination has been discussed in the literature, where the participation rates are exogenously specified. In this research, we investigate the channel coordination problem in which the members can decide on the values of participation rates. In a two-way subsidy strategy, the manufacturer shares the local advertising costs and retailers share the national advertising cost. Here, two scenarios are considered in which the participation rates can be either exogenously given or endogenously determined.

The remainder of the paper is organized as fol- lows. Section 2 briefly reviews the related literature. In Section 3, the notations and assumptions of the model are described. Then, in Section 4, cooperative benchmark solution (Section 4.1) and two other scenarios, including exogenous setting (Section 4.2) and endogenous setting (Section 4.3), are introduced. For each scenario, optimal advertising decisions considering collusion and Stackelberg cases are determined. Moreover, for each case in the second scenario, both oneway and two-way subsidy contracts are considered. In Section 5, the results are compared via theoretical and numerical analysis. Finally, conclusion and managerial implications are summarized in Section 6.

\section{Literature review}

Here, we briefly review the related researches. For an excellent review on cooperative advertising program, we refer the interested readers to Aust and Buscher [9] and J $\phi$ rgensen and Zaccour [21]. Among the earliest papers, in 1973, Berger analyzed cooperative advertising issue for the first time [8]. After that, other authors extended different aspects of Berger's work.

Some authors consider a case where there is more than one member in upstream and/or downstream of the channel and apply different mechanisms for channel coordination. Some consider one-manufacturer and two-retailers channel [22-25]; some study a channel with a single retailer and two competing suppliers [26]. However, the researches on competition between retailers or between suppliers are scarce.

In these studies, the effects of various competitive behaviors on cooperative advertising policies (i.e., competition on price or advertising plans) are investigated. For example, Chutani and Sethi [27] studied a supply chain with a manufacturer and two competing retailers. They assumed that retailers play a differential Nash game in choosing their strategies; Karray and Zaccour [28] studied a channel including two manufacturers and two retailers in which the national advertising efforts are specified as an exogenous parameter instead of decision variable; Zhang and Xie [29] studied a channel with multiple retailers for investigating the impact of the retailer's multiplicity on members' decision and total efficiency. In our research, one retailer's local advertising effort has a negative impact on another retailer's sale volume. Alaei et al. [30] studied a singleManufacturer-two-retailer supply chain where retailers compete for local advertising investment. They utilized Nash bargaining model in order to determine how the channel members must split the extra profit obtained by moving to the cooperation case.

There are a few researches in the literature that consider a two-way subsidy participation strategy, in which not only the manufacturer pays a fraction of the local advertising to retailers, but also retailers pay 
a part of the national advertising costs to the manufacturer. Kunter [13] considered a contract of royalty payments that can achieve the perfect coordination of the retailer-manufacturer channel. Their contract includes wholesale price, revenue sharing rate, and twoway subsidy participation rates. Zhang et al. [14] studied the impact of the reference price on decisions of members, and they applied the two-way subsidy participation where the participation rates are given. Ingene and Parry [22] considered channel coordination and examined the channel coordination by quantity discount and two-part tariff in a one-manufacturer and two-retailer channel. Lee \& Yang [31] used the same contracts in a one-retailer and two-competing-suppliers case. Bergen and John [32] examined the two-part tariff in a channel where two retailers and two suppliers compete. Zhang et al. [15] investigated the cooperative advertising problem with one retailer and one manufacturer under the bilateral participation strategy and concluded that it is necessary for participation rates to be given in order to achieve channel coordination.

\section{Notations and assumptions}

The following notations are used throughout the paper:

$\rho \quad$ Each retailer's dollar marginal profit per unit

$\rho^{\prime} \quad$ The manufacturer's dollar marginal profit per unit

$\varphi \quad$ The relative channel power of the manufacturer compared to each retailer

$S_{i} \quad$ Retailer $i$ 's market demand $(i=1,2)$

$d \quad$ Degree of substitutability between retailers

$b \quad$ Positive constant characterizing the market size

$N \quad$ The manufacturer's national advertising investment

$l_{i} \quad$ The retailer $i$ 's local advertising investment $(i=1,2)$

$\theta \quad$ The portion of the national investment paid by each retailer to the manufacturer

$\Theta \quad$ The fraction of the local advertising investment paid by the manufacturer to each retailer follows.

The assumptions of our proposed model are as

Assumption 1: The distribution channel includes one manufacturer and two retailers who compete in advertising plan.
We consider a one-manufacturer and two-retailer channel, in which the manufacturer $(M)$ sells the product to consumers through two retailers $\left(r_{1}\right.$ and $\left.r_{2}\right)$. All firms are willing to optimize their own revenue by choosing their local/national advertising investment as well as their participation rate on another firm's advertising plan.

Assumption 2: The manufacturer' and each retailer's dollar marginal profit per unit are $\rho^{\prime}>0$ and $\rho>0$, respectively, with $\varphi=\rho^{\prime} / \rho$.

We assume that both retailers have the same marginal profit. Note that the pricing decisions are not considered directly in our study and we mainly focus on advertising decisions. So, the retail and wholesale prices are exogenously determined. Note that $\varphi$ is the relative channel power of the manufacturer compared with each retailer.

Assumption 3: The market demand is simultaneously affected by the members' advertising efforts.

We assume that both retailers face similar market demand. Each retailer $i$ 's local advertising $\left(l_{i}\right)$ and manufacturer's national advertising $(N)$ have a positive effect on product sales of the retailer $i$. However, the rival retailer $j$ 's local advertising has a negative effect on retailer $i$ 's sale volume due to the competitive relationship between them. So, similar to Alaei et al. [30], we assume that the retailer $i$ 's demand function has the form $S_{i}=b\left(\sqrt{l_{i}}-d \sqrt{l_{j}}+\sqrt{N}\right),(0<d<$ $1 ; i=1,2 ; j=3-i)$, where $b$ is a positive constant characterizing the market size and $d$ represents the degree of substitutability between retailers. It can be denoted as the sensitivity of one retailer's market demand to change in other retailer's local advertising cost. Here, greater values of represent that the competition between retailers is fiercer. It is obvious that the market demand of retailer $i$ is increasing and concave function with respect to $l_{i}$ and $N$, but, it is decreasing and convex function with respect to $l_{j}$. Since, we have the following relations:

$$
\begin{aligned}
& \frac{\partial S_{i}}{\partial l_{i}}=\frac{b}{2 \sqrt{l_{i}}}>0, \quad \frac{\partial S_{i}}{\partial N}=\frac{b}{2 \sqrt{N}}>0, \\
& \frac{\partial S_{i}}{\partial l_{j}}=\frac{b d}{2 \sqrt{l_{i}}}<0, \quad \frac{\partial^{2} S_{i}}{\partial l_{i}^{2}}=-\frac{b}{4 \sqrt{l_{i}^{3}}}<0, \\
& \frac{\partial^{2} S_{i}}{\partial N^{2}}=-\frac{b}{4 \sqrt{N^{3}}}<0, \quad \frac{\partial^{2} S_{i}}{\partial l_{j}^{2}}=\frac{b d}{4 \sqrt{l_{j}^{3}}}>0 .
\end{aligned}
$$

Assumption 4: The local and national advertising investment allowances, $(\theta, \Theta)$, where $\theta \in[0,0.5]$ and $\Theta \in[0,1]$ can be either exogenously given (Scenario 1) or endogenously determined (Scenario 2). 
The parameter $\Theta$ is the fraction of the local advertising investment that the manufacturer agrees to share with each of the retailers. Similarly, $\theta$ is the portion of the national investment paid by each retailer to the manufacturer. Without loss of generality, we assume the same local advertising allowance for both retailers. Taking Assumptions 3 and 4 into account, the retailer $i$ 's, manufacturer's, sum of the retailers', and total channel's profit can be expressed, respectively, as follows:

$$
\begin{aligned}
& \Pi_{r_{i}}=b \rho\left(\sqrt{l_{i}}-d \sqrt{l_{j}}+\sqrt{N}\right)-\theta N-(1-\Theta) l_{i}, \\
& i=1,2, \quad j=3-i, \\
& \Pi_{M}=b \rho \varphi\left((1-d)\left(\sqrt{l_{1}}+\sqrt{l_{2}}\right)+2 \sqrt{N}\right) \\
& -(1-2 \theta) N-\Theta\left(l_{1}+l_{2}\right), \\
& \Pi_{r_{1}+r_{2}}=b \rho\left((1-d)\left(\sqrt{l_{1}}+\sqrt{l_{2}}\right)+2 \sqrt{N}\right) \\
& -2 \theta N-(1-\Theta)\left(l_{1}+l_{2}\right) \\
& \Pi_{S}=\Pi_{r_{1}}+\Pi_{r_{2}}+\Pi_{M}=b \rho(1+\varphi)\left(( 1 - d ) \left(\sqrt{l_{1}}\right.\right. \\
& \left.\left.+\sqrt{l_{2}}\right)+2 \sqrt{N}\right)-N-l_{1}-l_{2} .
\end{aligned}
$$

Assumption 5: (Game structure) Two scenarios including exogenous setting and endogenous setting are examined. For each scenario, two cases are investigated. The manufacturer always acts as the leader of Stackelberg game with the retailers being the followers. Moreover, the retailers can either compete (Stackelberg case) or cooperate (Collusion case).

Retailers cooperate in the collusion case in order to maximize their aggregate profit, while in the Stackelberg case, they play a Stackelberg game with the retailer 1 being the leader. For each case in the exogenous setting, we consider the conditions for perfect coordination. Also, we investigate the superiority of two-way subsidy strategy over the one-way subsidy strategy for each case in the endogenous setting.

Assumption 6: (Decision making) In the exogenous setting, the participation rates are exogenously given, and each member decides on his advertising investment. In the endogenous setting, the manufacturer optimizes participation rates; the retailer 1 decides on his local and the manufacturer's national advertising investment, while the retailer 2 only determines his local investment.

Moreover, in the endogenous setting, we examine a one-way subsidy strategy where the retailers' participation rate is assumed to be zero. So, the manufacturer only optimizes his participation rate. Note that each member cannot determine both his advertising investment and his participation rate in the endogenous setting, since this leads to trivial or unreasonable game results, as discussed in Zhang et al. [15]. For example, if one retailer is allowed to choose his local investment and his own participation rates, then his participation rate will be set to zero.

\section{Solution approaches}

In this section, we formulate the problem under the various settings. We examine the problem under two scenarios, where the participation rates can be either exogenously given or endogenously determined. The manufacturer leads the channel and Stackelberg game is played between the two echelons. For each scenario, two cases are investigated. In the first case, the retailers aim to optimize their aggregate profit; consequently, they follow collusion behavior, while the competition between retailers is based on Stackelberg game due to existence of a dominant retailer in the second case. We need the cooperative solution as a benchmark in order to examine the efficiency of different strategies.

\subsection{Cooperative solution}

When participation rates are either exogenously given or endogenously determined, we have the same solution for cooperative case. By solving the first order conditions $\partial \Pi_{S} / \partial l_{1}=0, \partial \Pi_{S} / \partial l_{2}=0$, and $\partial \Pi_{S} / \partial N=0$, the optimal cooperative solution is obtained as follows:

$$
\begin{aligned}
& l_{1}^{*}=l_{2}^{*}=\left[\frac{b \rho}{2}(1+\varphi)(1-d)\right]^{2}, \quad N^{*}=[b \rho(1+\varphi)]^{2}, \\
& \Pi_{S}=\frac{1}{2}(b \rho(1+\varphi))^{2}\left(2+(1-d)^{2}\right) .
\end{aligned}
$$

\subsection{Scenario 1: Exogenous setting}

Here, we consider a situation where the participation rates $(\theta, \Theta)$ are exogenously given. All the members of the channel know this and play a game in which the manufacturer first decides on national advertising cost, and then retailers choose their local advertising costs. We examine two decentralized cases and in both, the Stackelberg game is played between the two echelons with the manufacturer being the leader, and retailers choose their best responses according to the manufacturer's decision.

\subsubsection{Collusion case (the cooperating retailers)}

Here, retailers obey collusion behavior in downstream of the channel. So, the sum of their profit function should be maximized. They decide on their local advertising investments. By solving the first order conditions, $\partial \Pi_{r_{1}+r_{2}} / \partial l_{i}=0$ for $i=1,2$, we get $l_{1}=$ 
$l_{2}=(0.5 b \rho(1-d) /(1-\Theta))^{2}$. Then, the manufacturer takes the retailers' response into consideration and maximizes his profit by solving $\partial \Pi_{M} / \partial N=0$, which results in the national advertising cost to be equal to $N=(b \rho \varphi /(1-2 \theta))^{2}$.

\subsubsection{Stackelberg case (the dominant retailer)}

In this case, retailers play a Stackelberg game to determine their local advertising investments. The decision variables can be determined under the following sequence. First, retailer 2 determines $l_{2}$, then by substituting $l_{2}$ into Eq. (1), $l_{2}$ will be omitted. Now, retailer 1 decides on the amount of $l_{1}$, then the manufacturer's profit function will be simplified by substituting $l_{1}$ and $l_{2}$ into Eq. (2):

$$
\begin{aligned}
& l_{2} \in \arg \max _{l_{2}} \Pi_{r_{2}}\left(l_{1}, l_{2}, N, \theta, \Theta\right), \\
& l_{1} \in \arg \max _{l_{1}} \Pi_{r_{1}}\left(l_{1}, N, \theta, \Theta\right), \\
& N \in \arg \max _{N, \theta, \Theta} \Pi_{M}(N, \theta, \Theta) .
\end{aligned}
$$

The retailer 2's response can be obtained by solving $\partial \Pi_{r_{2}} / \partial l_{2}=0$, and we get $l_{2}=(0.5 b \rho /(1-\Theta))^{2}$. Then, the dominant retailer (retailer 1 ), knowing the retailer 2's response, maximizes his profit function by $\partial \Pi_{r_{1}} / \partial l_{1}=0$, which leads to the same value for his local investment cost as for retailer 2 . Now, substituting $l_{1}$ and $l_{2}$ into Eq. (2) and letting $\partial \Pi_{M} / \partial N=0$, the national advertising cost is obtained as $N=(b \rho \varphi /(1-$ $2 \theta))^{2}$.

It is obvious that both cases result in the same values for the decision variables. Now, by appropriately specifying the participation rates, bilateral strategy will lead to complete channel coordination, in which the whole channel's profit is maximized.

Proposition 1: When the participation rates are exogenously determined, the two-way subsidy strategy is capable of coordinating the channel if and only if they are specified as follows:

In the Collusion case:

$$
\Theta=\frac{\varphi}{1+\varphi}, \quad \theta=\frac{1}{2(1+\varphi)}
$$

In the Stackelberg case:

$$
\begin{aligned}
& \text { if } \varphi>d /(1-d) \text { : } \\
& \Theta=\frac{(1+\varphi)(1-d)-1}{(1+\varphi)}, \quad \theta=\frac{1}{2(1+\varphi)} .
\end{aligned}
$$

And, if $\varphi<d /(1-d)$, there is no feasible two-way subsidy strategy.
Proof: If $\varphi<d /(1-d)$, the optimal value of $\Theta$ will be equal to zero. So, we will have unilateral strategy. Now, consider that $\varphi>d /(1-d)$, the channel will be coordinated if and only if cooperative solution coincides with that of Collusion and Stackelberg solutions. Comparing $l_{i}^{*}$ with $l_{i}$ and $N^{*}$ with $N$, the proof will be completed. $\square$

Insight 1: It is obvious that as the degree of substitutability increases (or, in other words, retailers compete more fiercely), retailers' local investment and the manufacturer's participation will be decreased. However, retailers' participation in national advertising investment only depends on $\varphi$. As $\varphi$ increases, the manufacturer will prefer to share more of local advertising costs, however, the retailers will tend to share less of national advertising cost.

Insight 2: The manufacturer should set his profit margin greater than $d /(1-d)$ in order to have a feasible two-way subsidy strategy, and consequently, to achieve channel coordination.

Insight 3: The amount of subsidy paid by the manufacturer to the retailers in the Stackelberg case is greater than that in the Collusion case, while the amount of subsidy paid by the retailers to the manufacturer is the same in both cases.

The manufacturer pays an amount of $T=\Theta l_{i}$ to the retailer $i$. The value of $l_{i}$ is the same in both cases, while we always have $\Theta^{C n}>\Theta^{\text {stag }}$; so, we have $T^{C n}>T^{\text {stag }}$. Each retailer pays an amount of $T^{\prime}=\theta N$ to the manufacturer. Since $\theta$ and $N$ are the same in both cases, then $T^{\prime}$ is the same.

\subsection{Scenario 2: Endogenous setting}

4.3.1. Collusion case (the cooperating retailers)

Below, we discuss each of the one-way and two-way strategies:

(a) One-way subsidy strategy $(\theta=0)$. In this situation, first, the manufacturer chooses his national advertising cost $(N)$ as well as his participation rate $(\Theta)$, then the duopolistic retailers decide on their local advertising program. So, retailers' response is equal to $l_{1}=l_{2}=(b \rho(1-d) / 2(1-$ $\Theta))^{2}$ from $\partial \Pi_{r_{1}+r_{2}} / \partial l_{1}=0$ and $\partial \Pi_{r_{1}+r_{2}} / \partial l_{2}=$ 0 . Now, the manufacturer's problem can be rewritten as follows:

$$
\begin{aligned}
\Pi_{M}= & (b \rho(1-d))^{2}\left(\frac{\varphi}{1-\Theta}-\frac{\Theta}{2(1-\Theta)^{2}}\right) \\
& +2 b \rho \varphi \sqrt{N}-N .
\end{aligned}
$$

Then, by solving $\partial \Pi_{M} / \partial N=\partial \Pi_{M} / \partial \Theta=0$, the solution will be obtained as below: 


$$
\begin{aligned}
& \Theta=\frac{2 \varphi-1}{2 \varphi+1}, \quad N=(b \rho \varphi)^{2}, \\
& l_{1}=l_{2}=\left(\frac{b \rho(2 \varphi+1)(1-d)}{4}\right)^{2} .
\end{aligned}
$$

(b) Two-way subsidy strategy $(\theta \neq 0)$. Under this strategy, the manufacturer leads the game and decides on both participation rates, while the retailers act as followers and choose their local advertising costs as well as the manufacturer's advertising expenditure. The retailers' response will be $l_{1}=l_{2}=(b \rho(1-d) / 2(1-\Theta))^{2}$ and $N=$ $(b \rho / 2 \theta)^{2}$ from $\partial \Pi_{r_{1}+r_{2}} / \partial l_{1}=0, \partial \Pi_{r_{1}+r_{2}} / \partial l_{2}=0$ and $\Pi_{r_{1}+r_{2}} / \partial N=0$. Then, the manufacturer's problem can be rewritten as:

$$
\Pi_{M}=(b \rho)^{2}\left(\frac{\varphi}{\theta}-\frac{1-2 \theta}{4 \theta^{2}}+\frac{\varphi(1-d)^{2}}{1-\Theta}-\frac{\Theta(1-d)^{2}}{2(1-\Theta)^{2}}\right)_{(14}
$$

Now, by solving $\partial \Pi_{M} / \partial \theta=\partial \Pi_{M} / \partial \Theta=0$, we get the solution as follows:

$$
\begin{aligned}
& \Theta=\frac{2 \varphi-1}{2 \varphi+1}, \quad \theta=\frac{1}{2 \varphi+1}, \\
& N=\left(\frac{b \rho(2 \varphi+1)}{2}\right)^{2}, \\
& l_{1}=l_{2}=\left(\frac{b \rho(2 \varphi+1)(1-d)}{4}\right)^{2} .
\end{aligned}
$$

\subsubsection{Stackelberg case (the dominant retailer)}

Below we discuss each of the one-way and two-way strategies:

(a) One-way subsidy strategy. In this situation, first, the manufacturer chooses his national advertising $\operatorname{cost}(N)$ as well as his participation rate $(\Theta)$, then the duopolistic retailers decide on their local advertising program. First, the retailer 1 chooses his advertising level, then the retailer 2 chooses $l_{2}$. So, retailers' response is equal to $l_{1}=l_{2}=$ $(b \rho / 2(1-\Theta))^{2}$. Now, the manufacturer's problem can be rewritten as follows:

$$
\begin{aligned}
\Pi_{M}= & (b \rho)^{2}\left(\frac{\varphi(1-d)}{1-\Theta}-\frac{\Theta}{2(1-\Theta)^{2}}\right) \\
& +2 b \rho \varphi \sqrt{N}-N .
\end{aligned}
$$

Then, by solving $\partial \Pi_{M} / \partial N=\partial \Pi_{M} / \partial \Theta=0$, the solution will be obtained as:

$$
\begin{aligned}
& \Theta=\frac{2 \alpha-1}{2 \alpha+1}, \quad N=(b \rho \varphi)^{2}, \\
& l_{1}=l_{2}=\left(\frac{b \rho(2 \alpha+1)}{4}\right)^{2},
\end{aligned}
$$

where $\alpha=\varphi(1-d)$. (b) Two-way subsidy strategy. Under this strategy, the manufacturer leads the game and decides on both participation rates, while retailers act as followers and choose advertising costs. In the downstream echelon, the dominant retailer decides on his local and national advertising level, while the other one only chooses his own local investment cost.

Similar to the previous section, from the Stackelberg game between retailers, we have $l_{1}=$ $l_{2}=(b \rho / 2(1-\Theta))^{2}$ and $N=(b \rho)^{2} / 4 \theta^{2}$. Now, by substituting retailers' response into Eq. (2), the manufacturer's problem can be rewritten as follows:

$$
\Pi_{M}=(b \rho)^{2}\left(\frac{\varphi}{\theta}-\frac{1-2 \theta}{4 \theta^{2}}+\frac{\varphi(1-d)}{1-\Theta}-\frac{\Theta}{2(1-\Theta)^{2}}\right)
$$

By solving $\partial \Pi_{M} / \partial \theta=\partial \Pi_{M} / \partial \Theta=0$, we get the optimal values for participation rates. Substituting $\theta$ and $\Theta$ into retailers' response, the solution is obtained follows:

$$
\begin{aligned}
& \Theta=\frac{2 \alpha-1}{2 \alpha+1}, \quad \theta=\frac{1}{2 \varphi+1}, \\
& N=\left(\frac{b \rho(2 \varphi+1)^{2}}{2}\right) \\
& l_{1}=l_{2}=\left(\frac{b \rho(2 \alpha+1)}{4}\right)^{2},
\end{aligned}
$$

where $\alpha=\varphi(1-d)$.

\section{Computational results}

\subsection{Comparison between strategies and cases}

In this section, we examine the performance of different strategies under two cases discussed above. We summarize the members' decision and profit under different strategies in Tables 1 and 2. The solution of collusion case is shown in Table 1 and the Stackelberg case is summarized in Table 2. The second and third columns of each table pertain to one-way and two-way strategies, respectively. For both strategies of each case, the solution $\left(\Theta, \theta, N, l_{i}\right)$, the members' individual profit, and whole channel's profit are computed. It is obvious that none of the strategies can achieve the perfect coordination of the channel. It can be proved by comparing the whole channel's profit under cooperative case with that of each strategy. Note that in order to have a feasible solution at collusion and Stackelberg cases, we should have $\varphi>0.5$ and $\varphi>1 / 2(1-d)$, respectively. It can be proved by letting the value of $\Theta$ 
Table 1. Solution of collusion case under the one-way and two-way subsidy strategies if $\varphi>0.5$.

\begin{tabular}{lll}
\hline Comparison measure & \multicolumn{1}{c}{ One-way } & Two-way \\
\hline$\Theta$ & $\frac{2 \varphi-1}{2 \varphi+1}$ & $\frac{2 \varphi-1}{2 \varphi+1}$ \\
$\theta$ & 0 & $\frac{1}{2 \varphi+1}$ \\
$N\left(\times \frac{k}{4}\right)$ & $4 \varphi^{2}$ & $(2 \varphi+1)^{2}$ \\
$l_{i}\left(\times \frac{k}{16}\right)$ & $(2 \varphi+1)^{2}(1-d)^{2}$ & $(2 \varphi+1)^{2}(1-d)^{2}$ \\
$\Pi_{r_{i}}\left(\times \frac{k}{8}\right)$ & $(2 \varphi+1)(1-d)^{2}+8 \varphi$ & $(2 \varphi+1)\left(2+(1-d)^{2}\right)$ \\
$\Pi_{M}\left(\times \frac{k}{8}\right)$ & $(2 \varphi+1)^{2}(1-d)^{2}+8 \varphi^{2}$ & $(2 \varphi+1)^{2}\left(2+(1-d)^{2}\right)$ \\
$\Pi_{S}\left(\times \frac{k}{8}\right)$ & $(2 \varphi+1)(2 \varphi+3)(1-d)^{2}+8 \varphi(\varphi+2)$ & $(2 \varphi+1)(2 \varphi+3)\left(2+(1-d)^{2}\right)$ \\
\hline
\end{tabular}

Note: $k=(b \rho)^{2}$

Table 2. Solution of Stackelberg case under the one-way and two-way subsidy strategies if $\varphi>1 / 2(1-d)$.

\begin{tabular}{lll}
\hline Comparison measure & \multicolumn{1}{c}{ One-way } & Two-way \\
\hline$\Theta$ & $\frac{2 \alpha-1}{2 \alpha+1}$ & $\frac{2 \alpha-1}{2 \alpha+1}$ \\
$\theta$ & 0 & $\frac{1}{2 \varphi+1}$ \\
$N\left(\times \frac{k}{4}\right)$ & $4 \varphi^{2}$ & $(2 \varphi+1)^{2}$ \\
$l_{i}\left(\times \frac{k}{16}\right)$ & $(2 \alpha+1)^{2}$ & $(2 \alpha+1)$ \\
$\Pi_{r_{i}}\left(\times \frac{k}{8}\right)$ & $(2 \alpha+1)(1-2 d)^{2}+8 \varphi$ & $(2 \alpha+1)(1-2 d)+2(2 \varphi+1)$ \\
$\Pi_{M}\left(\times \frac{k}{8}\right)$ & $(2 \alpha+1)^{2}+8 \varphi^{2}$ & $(2 \alpha+1)^{2}+2(2 \varphi+1)^{2}$ \\
$\Pi_{S}\left(\times \frac{k}{8}\right)$ & $(2 \alpha+1)(2 \alpha+3-4 d)+8 \varphi(\varphi+2)$ & $(2 \alpha+1)(2 \alpha+3-4 d)+2(2 \varphi+1)(2 \varphi+3)$ \\
\hline
\end{tabular}

Note: $k=(b \rho)^{2}$ and $\alpha=\varphi(1-d)$

be positive. We define $\eta$ as the performance (efficiency) measure of each strategy as below:

$$
\eta=1-\frac{\pi_{s}^{C o}-\Pi_{s}^{i}}{\Pi_{s}^{C o}} .
$$

The value of $\eta$ corresponds to the ratio of each strategy's total profit to that of cooperative case. Table 3 summarizes the results.

Insight 4: The efficiency of the channel under Stackelberg case is greater than that of collusion case in both one-way and two-way subsidy strategies for $d<2 / 3$.

This result can be proved by considering the results of Table 3 . Figures 1 and 2 illustrate the
Table 3. The efficiency of the different cases and strategies for endogenous scenario.

\begin{tabular}{ll}
\hline Case (strategy) & Efficiency $(\boldsymbol{\eta})$ \\
\hline Collusion (one-way) & $1-\frac{2+0.25(1-d)^{2}}{(1+\varphi)^{2}\left(2+(1-d)^{2}\right)}$ \\
Collusion (two-way) & $1-\frac{1}{4(1+\varphi)^{2}}$ \\
Stackelberg (one-way) & $1-\frac{2+(0.5-d)^{2}}{(1+\varphi)^{2}\left(2+(1-d)^{2}\right)}$ \\
Stackelberg (two-way) & $1-\frac{0.5+(0.5-d)^{2}}{(1+\varphi)^{2}\left(2+(1-d)^{2}\right)}$
\end{tabular}




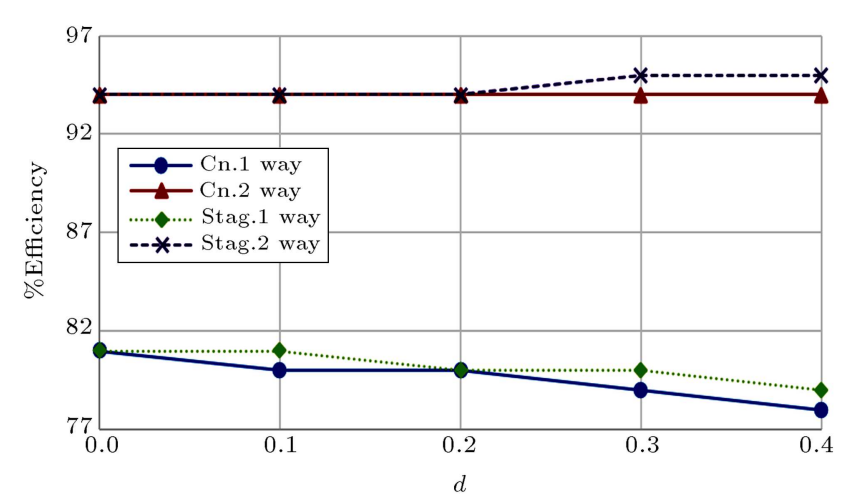

Figure 1. Channel efficiency with respect to $d$ for $\varphi=1$.

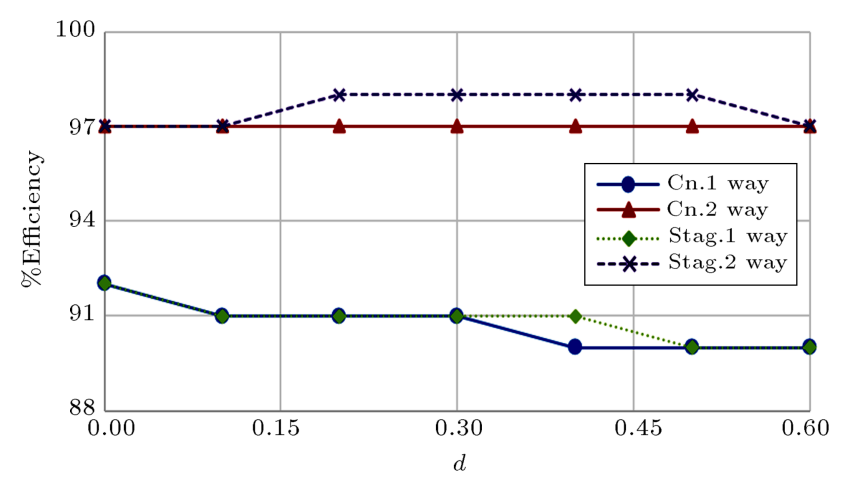

Figure 2. Channel efficiency with respect to $d$ for $\varphi=2$.

channel efficiency for $\varphi=1$ and $\varphi=2$, respectively.

Furthermore, it can be easily proved that efficiency of the two-way strategy is higher than that of the one-way strategy in both collusion and Stackelberg cases. By applying the two-way strategy rather than the one-way, the efficiency increases by $3 / 2(1+\varphi)^{2}(2+$ $\left.(1-d)^{2}\right)$ in both cases. So, we get the following insight.

Insight 5: The superiority of the two-way strategy depends neither on the values of $\varphi$ and $d$ nor on the behaviour which is obeyed by the retailers.

\subsection{Illustrative example}

In order to validate the model, we also present a numerical example by considering the parameter value as $b=1000, \rho=2, \varphi=1,2,3$, and $d=0.15,0.3,0.45,0.6$. The optimal values for collusion and Stackelberg cases are given in Table 4 and Table 5 , respectively. Note that the values of $N, l_{i}, \Pi_{r_{i}}, \Pi_{M}$, and $\Pi_{s}$ have been divided by 1000000 in both tables.

From Tables 4 and 5, note that by moving from one-way to two-way strategy, $\Theta$ and $l_{i}$ remain unchanged; the values of $N, \Pi_{M}$, and $\Pi_{s}$ are increased; however, $\Pi_{r_{i}}$ is decreased.

\section{Conclusion and managerial implications}

This paper investigated the cooperative advertising problem by taking the competition/cooperation effect of retailers into account. We applied game-theoretic approach in order to formulate the behavior among the members of the supply chain. We investigated the oneway subsidy strategy where the manufacturer shares a part of retailers' local advertising investment. A situation, where retailers also share the manufacturer's national advertising investment, was considered in the two-way subsidy strategy.

It was shown that: a) When the participation rates are exogenously given, the two-way subsidy strategy is capable of coordinating the channel regardless of which behavior is obeyed between retailers; b) In the endogenous setting, assuming each member aims to maximize his own profit, the perfect coordination will not be obtained. The efficiency of the two-way strategy is higher than that of the one-way strategy in both collusion and Stackelberg cases. By applying the two-

Table 4. The optimal values for collusion case.

\begin{tabular}{|c|c|c|c|c|c|c|c|c|c|c|c|c|c|c|c|}
\hline \multirow[b]{2}{*}{$\varphi$} & \multirow[b]{2}{*}{$d$} & \multicolumn{7}{|c|}{ One-way } & \multicolumn{7}{|c|}{ Two-way } \\
\hline & & $\Theta$ & $\theta$ & $N$ & $l_{i}$ & $\Pi_{r_{i}}$ & $\Pi_{M}$ & $\Pi_{S}$ & $\Theta$ & $\theta$ & $N$ & $l_{i}$ & $\Pi_{r_{i}}$ & $\Pi_{M}$ & $\Pi_{S}$ \\
\hline 1 & 0.15 & 0.33 & 0 & 4 & 1.6 & 5.1 & 7.3 & 17.4 & 0.33 & 0.33 & 9 & 1.6 & 4.1 & 12.3 & 20.4 \\
\hline 1 & 0.3 & 0.33 & 0 & 4 & 1.1 & 4.7 & 6.2 & 15.7 & 0.33 & 0.33 & 9 & 1.1 & 3.7 & 11.2 & 18.7 \\
\hline 1 & 0.45 & 0.33 & 0 & 4 & 0.7 & 4.5 & 5.4 & 14.3 & 0.33 & 0.33 & 9 & 0.7 & 3.5 & 10.4 & 17.3 \\
\hline 1 & 0.6 & 0.33 & 0 & 4 & 0.4 & 4.2 & 4.7 & 13.2 & 0.33 & 0.33 & 9 & 0.4 & 3.2 & 9.7 & 16.2 \\
\hline 2 & 0.15 & 0.60 & 0 & 16 & 4.5 & 9.8 & 25.0 & 44.6 & 0.60 & 0.20 & 25 & 4.5 & 6.8 & 34.0 & 47.6 \\
\hline 2 & 0.3 & 0.60 & 0 & 16 & 3.1 & 9.2 & 22.1 & 40.6 & 0.60 & 0.20 & 25 & 3.1 & 6.2 & 31.1 & 43.6 \\
\hline 2 & 0.45 & 0.60 & 0 & 16 & 1.9 & 8.8 & 19.8 & 37.3 & 0.60 & 0.20 & 25 & 1.9 & 5.8 & 28.8 & 40.3 \\
\hline 2 & 0.6 & 0.60 & 0 & 16 & 1.0 & 8.4 & 18.0 & 34.8 & 0.60 & 0.20 & 25 & 1.0 & 5.4 & 27.0 & 37.8 \\
\hline 3 & 0.15 & 0.71 & 0 & 36 & 8.9 & 14.5 & 53.7 & 82.8 & 0.71 & 0.14 & 49 & 8.9 & 9.5 & 66.7 & 85.8 \\
\hline 3 & 0.3 & 0.71 & 0 & 36 & 6.0 & 13.7 & 48.0 & 75.4 & 0.71 & 0.14 & 49 & 6.0 & 8.7 & 61.0 & 78.4 \\
\hline 3 & 0.45 & 0.71 & 0 & 36 & 3.7 & 13.1 & 43.4 & 69.5 & 0.71 & 0.14 & 49 & 3.7 & 8.1 & 56.4 & 72.5 \\
\hline 3 & 0.6 & 0.71 & 0 & 36 & 2.0 & 12.6 & 39.9 & 65.0 & 0.71 & 0.14 & 49 & 2.0 & 7.6 & 52.9 & 68.0 \\
\hline
\end{tabular}


Table 5. The optimal values for Stackelberg case.

\begin{tabular}{|c|c|c|c|c|c|c|c|c|c|c|c|c|c|c|c|}
\hline \multirow[b]{2}{*}{$\varphi$} & \multirow[b]{2}{*}{$d$} & \multicolumn{7}{|c|}{ One-way } & \multicolumn{7}{|c|}{ Two-way } \\
\hline & & $\Theta$ & $\theta$ & $N$ & $l_{i}$ & $\Pi_{r_{i}}$ & $\Pi_{M}$ & $\Pi_{S}$ & $\Theta$ & $\theta$ & $N$ & $l_{i}$ & $\Pi_{r_{i}}$ & $\Pi_{M}$ & $\Pi_{S}$ \\
\hline 1 & 0.15 & 0.26 & 0 & 4 & 1.8 & 4.9 & 7.6 & 17.5 & 0.26 & 0.33 & 9 & 1.8 & 3.9 & 12.6 & 20.5 \\
\hline 1 & 0.3 & 0.17 & 0 & 4 & 1.4 & 4.5 & 6.9 & 15.8 & 0.17 & 0.33 & 9 & 1.4 & 3.5 & 11.9 & 18.8 \\
\hline 1 & 0.45 & 0.05 & 0 & 4 & 1.1 & 4.1 & 6.2 & 14.4 & 0.05 & 0.33 & 9 & 1.1 & 3.1 & 11.2 & 17.4 \\
\hline 2 & 0.15 & 0.55 & 0 & 16 & 4.8 & 9.5 & 25.7 & 44.8 & 0.55 & 0.20 & 25 & 4.8 & 6.5 & 34.7 & 47.8 \\
\hline 2 & 0.3 & 0.47 & 0 & 16 & 3.6 & 8.8 & 23.2 & 40.7 & 0.47 & 0.20 & 25 & 3.6 & 5.8 & 32.2 & 43.7 \\
\hline 2 & 0.45 & 0.38 & 0 & 16 & 2.6 & 8.2 & 21.1 & 37.4 & 0.38 & 0.20 & 25 & 2.6 & 5.2 & 30.1 & 40.4 \\
\hline 2 & 0.6 & 0.23 & 0 & 16 & 1.7 & 7.7 & 19.4 & 34.9 & 0.23 & 0.20 & 25 & 1.7 & 4.7 & 28.4 & 37.9 \\
\hline 3 & 0.15 & 0.67 & 0 & 36 & 9.3 & 14.1 & 54.6 & 82.9 & 0.67 & 0.14 & 49 & 9.3 & 9.1 & 67.6 & 85.9 \\
\hline 3 & 0.3 & 0.62 & 0 & 36 & 6.8 & 13.0 & 49.5 & 75.6 & 0.62 & 0.14 & 49 & 6.8 & 8.0 & 62.5 & 78.6 \\
\hline 3 & 0.45 & 0.53 & 0 & 36 & 4.6 & 12.2 & 45.2 & 69.7 & 0.53 & 0.14 & 49 & 4.6 & 7.2 & 58.2 & 72.7 \\
\hline 3 & 0.6 & 0.41 & 0 & 36 & 2.9 & 11.7 & 41.8 & 65.1 & 0.41 & 0.14 & 49 & 2.9 & 6.7 & 54.8 & 68.1 \\
\hline
\end{tabular}

way strategy rather than the one-way, the efficiency (ratio of each strategy's total profit to that of the cooperative case) will be increased in both cases.

Our study can be extended to a case with multiple manufacturers and multiple retailers. One can adopt a more general demand function or stochastic demand function in order to generalize our finding regarding the benefits of exogenous setting compared to the endogenous setting and the advantages of two-way subsidy contract compared to the traditional one-way contract. Designing another contract with sufficient incentives for all members of the channel will be interesting. As previously discussed, the two-way subsidy strategy under collusion and Stackelberg cases is only feasible when specific conditions are met. Designating other contracts which make the two-way strategy always feasible is of absolute necessity.

\section{References}

1. Shin, H. and Tunca, T.I. "Do firms invest in forecasting efficiently? The effect of competition on demand forecast investment and supply chain coordination", Oper. Res., 58, pp. 1592-1610 (2010).

2. Kogan, K. and Tapiero, C. "Vertical pricing competition in supply chains: the effect of production experience and coordination", Int. Trans. Oper. Res., 15, pp. 461-479 (2008).

3. Alaei, S. and Setak, M. "Multi objective coordination of a supply chain with routing and service level consideration", Int. J. Prod. Econ., 167, pp. 271-281 (2015).

4. Alaei, S. and Khoshalhan, F. "A hybrid culturalharmony algorithm for multi-objective supply chain coordination", Sci. Iran. E, 22(3), pp. 1227-1241 (2015).

5. Li, X. and Wang, Q. "Coordination mechanisms of supply chain systems", Eur. J. Oper. Res., 179, pp. 1-16 (2007).
6. Darayi, M., Eskandari, H. and Geiger, C.D. "Supply chain channel coordination under sales rebate return policy contract using simulation optimization", Sci. Iran., 21(6), pp. 2295-2306 (2014).

7. Arshinder, K., Kanda, A. and Deshmukh, S.G. "A review on supply chain coordination: coordination mechanisms, managing uncertainty and research directions", In: Supply Chain Coordination Under Uncertainty, T.M. Choi, and T.C.E. Cheng, Edn., pp. 39-82, Berlin Heidelberg: Springer-Verlag (2011).

8. Berger, P.D. "Vertical cooperative advertising ventures", J. Market. Res., 9, pp. 309-312 (1972).

9. Aust, G. and Buscher, U. "Cooperative advertising models in supply chain management: A review", Eur. J. Oper. Res., 234, pp. 1-14 (2014).

10. Clark, S. "Dual destiny", Adweek, 41, pp. 60-66 (2000).

11. Brennan, L. "How retailers are putting it all together?", Sales Market. Manag., 5, pp. 62-65 (1988).

12. Lieb, R. "Co-op advertising: Digital's lost opportunity?", Report, Interactive Advertising Bureau, Local Search Association (2012).

13. Kunter, M. "Coordination via cost and revenue sharing in manufacturer-retailer channels", Eur. J. Oper. Res., 216, pp. 477-486 (2012).

14. Zhang, J., Gou, Q., Liang, L. and Huang, Z. "Supply chain coordination through cooperative advertising with reference price effect", Omega - Int. J. Manag. Sci., 41, pp. 345-353 (2013).

15. Zhang, J., Xie, J. and Chen, B. "Cooperative advertising with bilateral participation", Dec. Sci., 44, pp. 193-203 (2013).

16. Tsay, A.A. "The quantity flexibility contract and supplier-customer incentives", Manag. Sci., 45, pp. 1339-1358 (1999).

17. Zhang, H. and Zhong, S. "Co-op advertising analysis within a supply chain based on the product life cycle", rth Int. Conf. on Comput. Intel. and Sec. (CIS), pp. 1456-1460 (2011). 
18. Wang, S.D., Zhou, Y.W., Min, J. and Zhong, Y.G. "Coordination of cooperative advertising models in a one-manufacturer two-retailer supply chain system", Comput. Indust. Eng., 61(4), pp. 1053-1071 (2011).

19. Tsao, Y.C. and Sheen, G.J. "Effects of promotion cost sharing policy with the sales learning curve on supply chain coordination", Comput. Oper. Res., 39(8), pp. 1872-1878 (2012).

20. Ghadimi, S., Szidarovszky, F., Farahani, R.Z. and Khiabani, A.Y. "Coordination of advertising in supply chain management with cooperating manufacturer and retailers", IMA J. Manag. Math., 24(1), pp. 1-19 (2013).

21. J $\phi$ rgensen, S. and Zaccour, G. "A survey of gametheoretic models of cooperative advertising", Eur. J. Oper. Res., 237, pp. 1-14 (2014).

22. Ingene, C. and Parry, M. "Channel coordination when retailers compete", Market. Sci., 14, pp. 360-377 (1995).

23. Xiao, T., Yu, G., Sheng, Z. and Xia, Y. "Coordination of a supply chain with one-manufacturer and two-retailers under demand promotion and disruption management decisions", Ann. Oper. Res., 135, pp. 87109 (2005).

24. Xiao, T., Qi, X. and Yu, G. "Coordination of supply chain after demand disruptions when retailers compete", Int. J. Prod. Econ., 109, pp. 162-179 (2007).

25. Zhang, W.G., Fu, J., Li, H. and Xu, W. "Coordination of supply chain with a revenue-sharing contract under demand disruptions when retailers compete", Int. J. Prod. Econ., 138, pp. 68-75 (2012).

26. Choi, S. "Price competition in a channel structure with a common retailer", Market. Sci., 10, pp. 271296 (1991).

27. Chutani, A. and Sethi, S.P. "Optimal advertising and pricing in a dynamic durable goods supply chain", $J$. Optimiz. Theory App., 154, pp. 615-643 (2012).

28. Karray, S. and Zaccour, G. "Effectiveness of coop advertising programs in competitive distribution channels", Int. Game Theory Rev., 9, pp. 151-67 (2007).

29. Zhang, J. and Xie, J. "A game theoretical study of cooperative advertising with multiple retailers in a distribution channel", J. Sys. Sci. Sys. Eng., 21, pp. 37-55 (2012).

30. Alaei, S., Alaei, R. and Salimi, P. "A game theoretical study of cooperative advertising in a singlemanufacturer-two- retailers supply chain", Int. J. Adv. Manuf. Technol., 74, pp. 101-111 (2014).

31. Lee, C.Y. and Yang, R. "Supply chain contracting with competing suppliers under asymmetric information", IIE Trans., 45, pp. 25-52 (2013).
32. Bergen, M. and John, G. "Understanding cooperative advertising participation rates in conventional channels", J. Market. Res., 34(3), pp. 357-369 (1997).

\section{Appendix}

\section{Proof for Section 4.1}

In order to prove that the objective function is concave with respect to $N, l_{1}$, and $l_{2}$, we need to prove that the Hessian matrix is negative definite. The Hessian matrix is as follows:

$$
H=\left[\begin{array}{ccc}
H_{11} & 0 & 0 \\
0 & H_{22} & 0 \\
0 & 0 & H_{33}
\end{array}\right],
$$

where:

$$
\begin{aligned}
& H_{11}=\frac{\partial^{2} \Pi_{s}}{\partial l_{1}^{2}}=\frac{b \rho(1+\varphi)(1-d)}{-4 \sqrt{l_{1}^{3}}}, \\
& H_{22}=\frac{\partial^{2} \Pi_{s}}{\partial l_{2}^{2}}=\frac{b \rho(1+\varphi)(1-d)}{-4 \sqrt{l_{2}^{3}}} \\
& H_{33}=\frac{\partial^{2} \Pi_{s}}{\partial N^{2}}=\frac{b \rho(1+\varphi)}{-2 \sqrt{N^{3}}}
\end{aligned}
$$

It is obvious that $H_{11}<0, H_{11} H_{22}>0$ and $H_{11} H_{22} H_{33}<0$; so, the Hessian matrix is negative definite. Accordingly, the objective function is concave with respect to $N, l_{1}$, and $l_{2}$.

\section{Proof for Section 4.3.1-(a)}

The Hessian matrix of the objective function is as follows:

$$
H=\left[\begin{array}{cc}
H_{11} & 0 \\
0 & H_{22}
\end{array}\right]
$$

where:

$$
\begin{aligned}
& H_{11}=\frac{\partial^{2} \Pi_{M}}{\partial N^{2}}=\frac{-b \rho \varphi}{2 \sqrt{N^{3}}}, \\
& H_{22}=\frac{\partial^{2} \Pi_{M}}{\partial \Theta^{2}}=\frac{(b \rho(1-d))^{2}}{(1-\Theta)^{3}}\left(2 \varphi-\frac{\Theta+2}{1-\Theta}\right) .
\end{aligned}
$$

Since only the major diameter of the matrix is nonzero, the proof will be completed if we prove that each diagonal element is negative. $H_{11}$ is negative, however, $\mathrm{H}_{22}$ is negative under the following condition:

$$
\Theta>\frac{2 \varphi-2}{2 \varphi+1} \text {. }
$$

It is obvious that $\Theta^{*}$ meets the condition. Therefore, even though the concavity of the manufacturer's objective function could not be proved with certainty, as has been proved, the function is concave around the optimal solution. 
Proof for Section 4.3.1-(b)

Similar to the previous section, the Hessian matrix is:

$$
H=\left[\begin{array}{cc}
H_{11} & 0 \\
0 & H_{22}
\end{array}\right]
$$

where:

$$
\begin{aligned}
& H_{11}=\frac{\partial^{2} \Pi_{M}}{\partial \theta^{2}}=\frac{(b \rho)^{2}}{2 \theta^{4}}((4 \varphi+2) \theta-3), \\
& H_{22}=\frac{\partial^{2} \Pi_{M}}{\partial \theta^{2}}=\frac{(b \rho(1-d))^{2}}{(1-\Theta)^{3}}\left(2 \varphi-\frac{\Theta+2}{1-\Theta}\right) .
\end{aligned}
$$

$H_{11}$ and $H_{22}$, respectively, are negative under the following conditions:

$$
\begin{aligned}
& \theta<\frac{3}{2(2 \varphi+1)}, \\
& \Theta>\frac{2 \varphi-2}{2 \varphi+1} .
\end{aligned}
$$

It is easy to see that $\theta^{*}$ and $\Theta^{*}$ meet the conditions. So, the manufacturer's objective function is concave with respect to $\theta$ and $\Theta$ around the optimal solution.

Proof for Section 4.3.2-(a)

The Hessian matrix is:

$$
H=\left[\begin{array}{cc}
H_{11} & 0 \\
0 & H_{22}
\end{array}\right]
$$

where:

$$
\begin{aligned}
& H_{11}=\frac{\partial^{2} \Pi_{M}}{\partial N^{2}}=\frac{-b \rho \varphi}{2 \sqrt{N^{3}}}, \\
& H_{22}=\frac{\partial^{2} \Pi_{M}}{\partial \Theta^{2}}=\frac{(b \rho)^{2}}{(1-\Theta)^{3}}\left(2 \alpha-\frac{\Theta+2}{1-\Theta}\right) .
\end{aligned}
$$

$H_{11}$ is negative, however, $H_{22}$ is negative under the following condition:

$$
\Theta>\frac{2 \alpha-2}{2 \alpha+1} .
$$

The manufacturer's objective function is concave with respect to $N$ and $\Theta$ around the optimal solution, since $\Theta^{*}$ meets the condition.
Proof for Section 4.3.2-(b)

The Hessian matrix for the manufacturer's function is:

$$
H=\left[\begin{array}{cc}
H_{11} & 0 \\
0 & H_{22}
\end{array}\right],
$$

where:

$$
\begin{aligned}
& H_{11}=\frac{\partial^{2} \Pi_{M}}{\partial \theta^{2}}=\frac{(b \rho)^{2}}{2 \theta^{4}}((4 \varphi+2) \theta-3), \\
& H_{22}=\frac{\partial^{2} \Pi_{M}}{\partial \Theta^{2}}=\frac{(b \rho)^{2}}{(1-\Theta)^{3}}\left(2(\alpha-1)-\frac{3 \Theta}{1-\Theta}\right) .
\end{aligned}
$$

Here, $H_{11}$ and $H_{22}$ are negative under the following conditions, respectively:

$$
\begin{aligned}
& \theta<\frac{3}{2(2 \alpha+1)}, \\
& \Theta>\frac{2 \alpha-2}{2 \varphi+1},
\end{aligned}
$$

where $\theta^{*}$ and $\Theta^{*}$ satisfy the conditions. So, the function is concave with respect to $\theta$ and $\Theta$ around the optimal solution.

\section{Biographies}

Saeed Alaei obtained BS and MS degrees in Industrial Engineering from Amirkabir University of Technology, Tehran, Iran, in 2009, and Sharif University of Technology, Tehran, Iran, in 2011, respectively. He is currently a $\mathrm{PhD}$ degree candidate in Industrial Engineering at K.N. Toosi University of Technology. His research interests are mainly focused on supply chain coordination, multi-echelon inventory management, and game theory. He has authored papers published in national and international journals.

Mostafa Setak received BS, MS and PhD degrees in Industrial Engineering from Sharif University of Technology, Tehran, Iran; University of Science and Technology, Tehran, Iran; and Tarbiat Modares University, Tehran, Iran, respectively. He is currently an Associate Professor in the Faculty of Industrial Engineering at K.N. Toosi University of Technology. His research interests include vehicle routing problems, supply chain management, and supply chain coordination. He has authored numerous papers presented at conferences and published in national and international journals. 